\title{
Adolescents’ Knowledge of Diet-Related Chronic Diseases and Dietary Practices in Ghana
}

\author{
Christina A. Nti, Augustina Brown, Angelina Danquah
}

Department of Family and Consumer Sciences, School of Agriculture, University of Ghana, Legon, Ghana.

Email: cnti@ug.edu.gh

Received August $30^{\text {th }}, 2012$; revised September $30^{\text {th }}$, 2012; accepted October $7^{\text {th }}, 2012$

\begin{abstract}
Diet-related chronic diseases constitute public health and developmental challenges in Ghana. The Ghana Health Service in 2007 reported a national prevalence of diabetes of $11.6 \%$, 27.8\% for hypertension and $25 \%$ of women were reported to be overweight. Adolescents usually adopt lifestyles that negatively affect their nutritional and health status and increase their risk for development of diet-related chronic diseases later in life. The study was therefore carried out to investigate adolescents' knowledge of diet-related chronic diseases and its influence on their dietary practices. The study design was a cross-sectional survey involving 313 adolescents aged 14 - 18 years from public and private senior high schools. Structured interviews and diet assessment methods were used to collect information on respondents' knowledge of diet-related chronic diseases and dietary practices. The data collected were analyzed using the SPSS program version 16. The Chi-square test and ANOVA were used to determine the relationships between respondents' knowledge of diet-related chronic diseases and dietary practices. The results revealed that knowledge of diabetes, hypertension and obesity were low among the respondents with most of them (89.2\%) having fair to poor knowledge of the diseases. Eighty-eight percent of the adolescents ate three or more times in a day. Meals mostly skipped were breakfast and lunch. Dietary diversity of the respondents was generally poor. There was a significant relationship between knowledge of diet-related chronic diseases and the dietary practices of the adolescents. As knowledge of the diseases increased, dietary diversity also improved.
\end{abstract}

Keywords: Adolescents; Diet-Related Chronic Diseases; Dietary Practices

\section{Introduction}

Diet-related chronic diseases are long-term diseases that are not contagious and are largely preventable. They are diseases that result from poor eating habits. Examples include obesity, diabetes, cardiovascular diseases, cancer, osteoporosis, and dental diseases [1]. Diet-related chronic diseases require extensive care because most chronic diseases cannot be cured completely. Thus, people with chronic diseases may experience a lifetime discomfort, frequent doctor's visits, medical tests, medications, therapies and sometimes surgery. According to World Health Organization [2], diet-related chronic diseases are now the major cause of death and disability worldwide. Cardiovascular diseases, diabetes, obesity, cancer and respiratory diseases, account for $59 \%$ of the 57 million deaths annually and $46 \%$ of the global burden of disease. A relationship exists between dietary habits and diet-related chronic diseases. A change in dietary habits and physical activity has been reported to have a major impact in reducing the rates of these diseases, often in a relatively short time. At the moment, advances in medicine and rehabilitation have made it possible for some chronic diseases sufferers to manage the disease without affecting other areas of their lives [2].

In Ghana diet-related chronic diseases constitute public health and developmental challenges requiring the same intellectual and financial commitment afforded to infectious diseases such as malaria. The Ghana Demographic and Health Survey [3] indicated that the national prevalence of diabetes was $11.6 \%$. The survey recorded a national prevalence of $27.8 \%$ for hypertension. The report also indicated that $30 \%$ of women in Ghana were overweight and 9\% obese. A regional comparison showed that $20 \%$ of women in the Upper West, Northern and Upper East regions (the three northern regions) were overweight/obese while women of the Greater Accra region had the highest proportion of $45 \%$.

Adolescence is a developmental transition between childhood and adulthood that entails major interrelated physical, cognitive, and psychosocial changes in a person [4]. It is the second most critical period of physical growth in the life cycle after the first year and $25 \%$ of adult height is attained during this period. Nutritional 
needs are high for both male and female adolescents. The incidence of dietary inadequacies in adolescence is high. Substantial development and rates of growth combined with developmentally appropriate psychosocial changes, such as an increasing need for independence and a desire to make lifestyle choices that conform to peer ideals and differ from those of the family, place adolescents at risk of poor nutritional status [1]. Eating patterns established during adolescence may remain throughout the life cycle [5]. Adolescents increasingly seem to adopt lifestyles that negatively affect their nutritional and health status and therefore substantially increase their risk for premature development of diet-related diseases like cardiovascular diseases, diabetes and certain cancers. Poor dietary practices, sedentary leisure time spending and lack of physical activity are lifestyles that once instilled have a strong tendency to track from adolescence into adulthood and they become extremely resistant to modifications [6].

Adolescence is a unique intervention point in the life cycle. It offers a chance to acquire knowledge about optimal nutrition that could prevent, delay or hasten adultonset of diet-related chronic illnesses later in life. Understanding and promoting nutritional health, with appropriate assistance to adolescents, will help them improve their dietary habits and avoid diet-related chronic diseases such as diabetes, hypertension and obesity later in life. Since students in Day Second Cycle Institutions in Ghana are mainly adolescents who have much control over their dietary intakes, it is important to gain insight into their dietary habits, so as to offer appropriate interventions, to help them maintain optimal nutrition and health. This study, therefore, sought to assess adolescents' knowledge of diet-related chronic diseases and its influence on their dietary practices. It was hypothesized that there was no relationship between knowledge of diet-related chronic diseases and the dietary practices of day SHS students.

\section{Methodology}

\subsection{Study Design, Location and Population}

A cross-sectional survey was used to investigate the influence of knowledge of diet-related chronic diseases on the dietary practices of Senior High School students in the Ghana. The study was conducted in the Ga-East Municipality of the Greater Accra Region of Ghana. The population comprised all day students in their second year in senior high schools who were within the ages of 14 - 18 years. First year students were not included in the study because they had just been admitted and were still adjusting to the senior high school curriculum. Third year students were also not included because they were preparing for their final West Africa Senior Secondary School Certificate Examination (WASSSCE).

\subsection{Sample and Sampling Technique}

In order to have a fair representation of all adolescents in the senior high schools in the municipality, 4 schools were selected using purposive sampling techniques based on their location in the Municipality. The study schools purposively selected were West Africa Senior High located in the east, Action Senior High located in the south, Pre-Modal Senior High located in the west and Elim Senior High located in the northern part of the municipality. The total number of students who qualified for the study and were willing to participate were 940 . To determine the sampling frame $(k)$, the method $N / n=k$, where $\mathrm{N}=$ Total population and $\mathrm{n}=$ sample size was used $(k=940 / 313)$. With a sampling frame of 3 , a systematic random sampling technique was used to select every other $3^{\text {rd }}$ person on each school's list. Respondents who qualified for the study at the West Africa Senior High School, a public school, were 535. Out of this number, 178 were selected for the study. Students selected from the 3 private schools were 135. At Pre-Modal Senior High 150 qualified for the study and 50 were selected. At Action Senior High 180 qualified for the study and 60 were selected and at Elim Senior High 75 qualified for the study, of which 25 were selected for the study.

\subsection{Instruments and Data Collection}

The instruments used for data collection included a structured questionnaire and a food frequency questionnaire. A structured questionnaire was used to collect information on the background characteristics of respondents as well as their knowledge of diet-related chronic diseases and nutrition. A food frequency questionnaire consisting of food items from the 6 food groups in Ghana was used to assess frequency of consumption and dietary diversity of respondents. The food groups were starchy roots and plantain; cereals and grains; animal products; beans, nuts and oily seeds; fruits and vegetables; fats and oils. Respondents were required to tick the frequency of consumption of foods; whether on daily or weekly basis, occasionally or never. The data were collected between 2nd December, 2009 and $1^{\text {st }}$ March 2010.

\subsection{Data Analysis}

\subsubsection{Knowledge of Diseases}

Knowledge of hypertension, diabetes and obesity were scored using a scoring system developed by the researchers (Table 1). The scoring was based on the responses for the definition, causes and prevention of each of the diseases assessed, namely hypertension, diabetes and obesity. A correct answer attracted 1 mark while an incorrect answer attracted zero.

Marks awarded for answering all questions correctly 
Table 1. Scoring of knowledge of diet-related chronic diseases based on responses to questions related to the diseases.

\begin{tabular}{llc}
\hline Questions & Reponses & Score \\
\hline How do you understand the following: & Do not know & 0 \\
1. Diabetes & Related to sugar and energy loss & 1 \\
2. Hypertension & Related to high blood pressure. & 1 \\
3. Obesity & Related to overweight and excess fat in the body. & 1 \\
What are the causes of the diseases above? & Do not know & 1 \\
1. Diabetes & Related to too much sugar and carbohydrate intake. & 1 \\
2. Hypertension & Related to too much fat and salt intake. & 1 \\
3. Obesity & Related to binge eating and lack of exercise. & 0 \\
How can these diseases be prevented? & Do not know & 1 \\
1.Diabetes & Related to reduced sugar and carbohydrate intake & 1 \\
2. Hypertension & Related to reduced fat and salt intake & 1 \\
3. Obesity & Related to taking just enough food and exercising & \\
\hline
\end{tabular}

on each disease was 3. Scoring and rating was as follows: 0 - 1 = Poor knowledge; 2 = Fair knowledge; 3 = Good knowledge. Knowledge of the three diseases was scored by adding all the responses together. The highest mark for the overall knowledge of the 3 diseases was 9. Individuals with scores between zero and 3 were classified as having poor knowledge; those with scores between 4 and 6 as having fair knowledge, and those with scores of 7 - 9 as having good knowledge of the diseases.

\subsubsection{Dietary Data}

Dietary diversity scores were generated from the food frequency questionnaire. The scores gave an indication of the number of different food groups that each respondent consumed throughout the day, thus, a picture of the actual diversity of the meal consumed by respondents. The highest attainable score by each respondent based on all food groups consumed was 6 . Respondents with mean dietary diversity between 0 and 2 were classified as having low dietary diversity, 3 - 4 as fair diversity and 5 - 6 were classified as having high dietary diversity. All data collected were analyzed using The Statistical Package for Social Sciences (SPSS version 16.0). The chi-square test was used to ascertain the relationship between the knowledge of diet-related chronic diseases and the dietary practices of the high school students. Differences and associations were considered statistically significant for values of $\mathrm{P}<0.05$.

\subsection{Ethical Consideration}

Ethical approval was obtained from the Institutional Review Board of the Noguchi Memorial Institute for Medical Research, University of Ghana, Legon (Ethical Identification Number NMIMR-IRB CPN 003/10-11). Informed Consent was also obtained from the Heads and students of participating schools, after the purpose and significance of the study had been fully explained to them.

\section{Results and Discussion}

\subsection{Background Characteristics of Respondents}

The ages of the respondents ranged from 14 to 18 years. The mean ages for males and females were 16 years and 15 years respectively. Out of the 313 respondents, 52.1\% of respondents' parents worked in the private sector while the rest worked in the public sector. Major occupations of the parents included trading, banking, farming, teaching, nursing and social work. Even though it was not possible to document the financial capacities of respondents' parents in terms of income, the occupational background gives a general view that their parents' income cut across all the three economic levels; low, medium and high income earners.

\subsection{Knowledge of Diet-Related Chronic Diseases}

Table 2 shows respondents' level of knowledge of the three diet-related chronic diseases. More than half (63.6\%) of the respondents had poor knowledge of diabetes. This means that they did not have any understanding of the disease, did not know about its causes or prevention. Only $9.3 \%$ of the respondents had good knowledge of the disease. Data on the national prevalence of diabetes presented by the Ghana Demographic and Health Survey report [3] was 11.6\%. Figures from the Centre for Disease Control and Prevention at the Korle-bu Teaching Hospital in Accra also showed that 1080 and 1055 adolescents were diagnosed with diabetes in 2008 and 2009, respectively. It is possible that with such prevalence, most of the respondents would not have heard any discussions about the disease. The results therefore imply that either the Regenerative Health Programme initiated by the Ghana Health Service to educate people on some of these chronic diseases is not empha- 
Table 2. Respondents' level of knowledge of diabetes by sex.

\begin{tabular}{ccccc}
\hline \multirow{2}{*}{$\begin{array}{c}\text { Level of knowledge } \\
\text { on diabetes }\end{array}$} & \multicolumn{2}{c}{ Male } & \multicolumn{2}{c}{ Female } \\
\cline { 2 - 5 } & Number & $\%$ & Number & $\%$ \\
\hline Good & 15 & 4.8 & 14 & 4.5 \\
Fair & 46 & 14.7 & 39 & 12.5 \\
Poor & 93 & 29.7 & 106 & 33.9 \\
Total & 154 & 49.1 & 159 & 50.9 \\
\hline
\end{tabular}

$x^{2}=1.799$, P-value $=0.615$.

sizing diabetes enough or students have not been educated enough on diabetes through the programme. The level of knowledge of diabetes among males was slightly higher than that of the females but the difference was not statistically significant, indicating that gender does not predict respondents' level of knowledge of diabetes.

\subsection{Respondents' Level of Knowledge of Hypertension}

Respondents' level of knowledge of hypertension by gender is presented in Table 3. About half of the respondents (50.5\%) had poor knowledge of hypertension. Most of the respondents defined the disease as a disease of "more blood". Only 29.7\% had good knowledge of the disease. The poor knowledge of the disease could be attributed to the local dialect translation of hypertension. In the local "Twi" dialect, hypertension is named as "mogyammoroso" which literally means "excess blood". Thus, not only is such understanding of the disease wrong, it also explains why the students could not identify its causes or prevention. According to the Ghana Demographic and Health Survey report [3], the national prevalence for hypertension was $27.8 \%$, indicating that nearly one out of every three adults in the country was affected by the disease. In the Greater Accra region, the prevalence rate was $37 \%$. With half of the respondents having poor knowledge of the disease, hypertension may continue to increase due to unhealthy lifestyles, changes in eating patterns, increased consumption of fast foods, pre-prepared meals and carbonated drinks among respondents. World Health Organization [1] projected that diet-related chronic diseases will still occupy a critically important position up to the year 2020. This should serve as a wake-up call to health practitioners and service providers in Ghana to educate more people especially the youth, about the causes and prevention of some of these diseases. No significant differences were observed between males and females in terms of level of knowledge of hypertension.

\subsection{Respondents' Level of Knowledge of Obesity}

More than half of the respondents (63.6\%) had poor knowledge of obesity (Table 4). Less than a third of the
Table 3. Respondents’ knowledge of hypertension by sex.

\begin{tabular}{ccccc}
\hline \multirow{2}{*}{$\begin{array}{c}\text { Level of knowledge } \\
\text { on hypertension }\end{array}$} & \multicolumn{2}{c}{ Male } & \multicolumn{2}{c}{ Female } \\
\cline { 2 - 5 } & Number & $\%$ & Number & $\%$ \\
\hline Good & 46 & 14.7 & 47 & 15 \\
Fair & 33 & 10.5 & 29 & 9.3 \\
Poor & 75 & 24.0 & 83 & 26.5 \\
Total & 154 & 49.1 & 159 & 50.9 \\
\hline
\end{tabular}

$x^{2}=6.271$, P-value $=0.099$

Table 4. Respondents’ knowledge of obesity by sex.

\begin{tabular}{ccccc}
\hline \multirow{2}{*}{$\begin{array}{c}\text { Level of knowledge } \\
\text { on obesity }\end{array}$} & \multicolumn{2}{c}{ Male } & \multicolumn{2}{c}{ Female } \\
\cline { 2 - 5 } & Number & $\%$ & Number & $\%$ \\
\hline Good & 15 & 4.8 & 14 & 4.5 \\
Fair & 46 & 14.7 & 39 & 12.5 \\
Poor & 93 & 29.7 & 106 & 33.9 \\
Total & 154 & 49.1 & 159 & 50.9 \\
\hline
\end{tabular}

$x^{2}=1.799, \mathrm{P}=0.615$.

respondents had fair knowledge while a few (9.3\%) had good knowledge of the disease. Lack of knowledge of the disease could be attributed to the fact that in some communities, people do not view obesity as a disease. People with such physical traits are rather perceived as living well and so it is less likely for such persons to report to hospital to seek help. This is confirmed by figures at the Disease Control Unit of the Korle-bu Teaching Hospital, which indicated that no adolescent reported to the hospital in 2007 and 2008, respectively, with the problem of obesity [7]. This raises the need to educate the adolescents on lifestyle choices that lead to obesity. Again, the level of knowledge of obesity among the males and females was not statistically significant. Sources of information on the diseases by respondents who had some knowledge included listening to talk shows (33.3\%), friends and relatives (31.3\%), reading about the diseases on their own (28.9\%) and news broadcast (21.2\%).

\subsection{Dietary Practices of Respondents}

\subsubsection{Number of Times Respondents Ate Daily}

A greater proportion of the respondents (87.9\%) ate three times or more in a day. This pattern of consumption is in agreement with WHO recommendation of three meals a day. The rest ate once $(1.6 \%)$ or twice $(10.5 \%)$ in a day. Other studies among adolescent secondary students in Ghana have shown $83 \%$ and $84 \%$ of the respondents eating three times a day respectively $[8,9]$. Lansah [10] in a study of adolescent food habits in Koforidua also found $53 \%$ of adolescents eating three times a day. 


\subsubsection{Meals Frequently Skipped by Respondents}

In terms of meals frequently skipped by respondents, the results in Figure 1 show that $41.8 \%$ of the respondents skipped breakfast. This was not very encouraging because breakfast is very important especially among adolescents who are still in school. Breakfast is considered important because it has a role in alleviating short-term hunger thereby improving cognition, short term memory and concentration [11]. Lunch was skipped by $16 \%$ of the respondents. As with breakfast, eating lunch increases the intakes of energy, protein and other nutrients. The high intake of lunch among the respondents could be attributed to the fact that, most of them were given money to buy food at school. This finding is contrary to the study of Gleason and Suitor [12] that found that almost $25 \%$ of adolescents skipped lunch in the United States. Only a few of the respondents skipped supper. This finding is similar to a study by Lin et al. [13], on family meals in the United States. According to them, dinner was the most frequently consumed meal among adolescents. Another study conducted by Kyei-Baffour [14] in the Atwima-Nwabiagya district of the Ashanti Region, also in Ghana, reported that the most important meal to children aged 9 - 17 years was supper.

\subsubsection{Respondents' Reasons for Skipping Meals}

A number of reasons were given by the respondents for skipping meals. Almost all the respondents (94\%) who skipped breakfast did so due to lack of time; either they woke up late, or they were late for school. A few (4\%) indicated that they felt uncomfortable after eating breakfast so they had decided to stop eating breakfast. The reason attributed to skipping supper was intake of heavy lunch and/or eating lunch close to supper time. According to Story and Stang [15], meal skipping is common among adolescents especially during middle and late adolescence. They reported that breakfast was the most commonly skipped meal and was attributed to lack of time, desire to sleep longer and lack of appetite. They observed that adolescents who skipped meals had lower total daily energy, vitamin and mineral intakes compared to those who did not skip meals.

\subsubsection{Diversity of Respondents' Diet}

Figure 2 shows the respondents dietary diversity. Foods mostly consumed were cereals and cereal products. Most vegetables were consumed mainly in stews and soups with fish, meat or poultry. The consumption of fruits was low. Respondents' dietary diversity was generally low, with half of them (56\%) having low dietary diversity scores (DDS). A poor diet is a risk factor for the major chronic diseases that are leading causes of ill-health such as hypertension, diabetes, heart diseases and some forms of cancer [16]. A significant association was observed between knowledge of diseases and dietary diversity scores. As knowledge of the diseases increased, dietary practices also improved (Table 5). Respondents who had good knowledge of the diseases had better dietary diversity.

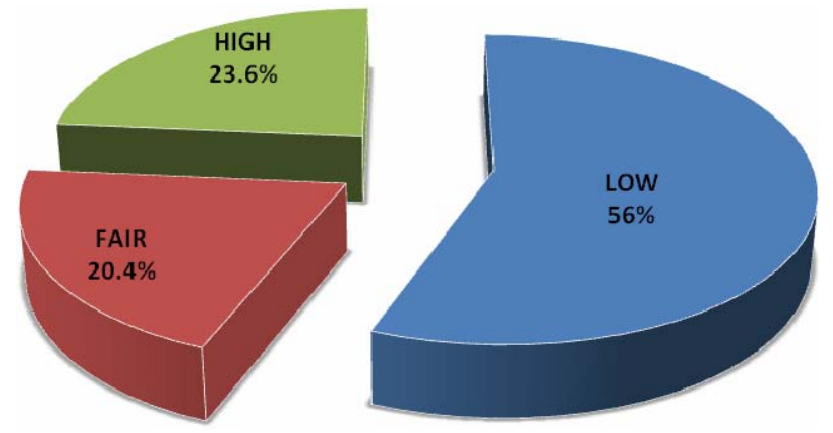

Figure 2. Diversity of respondents' diet.

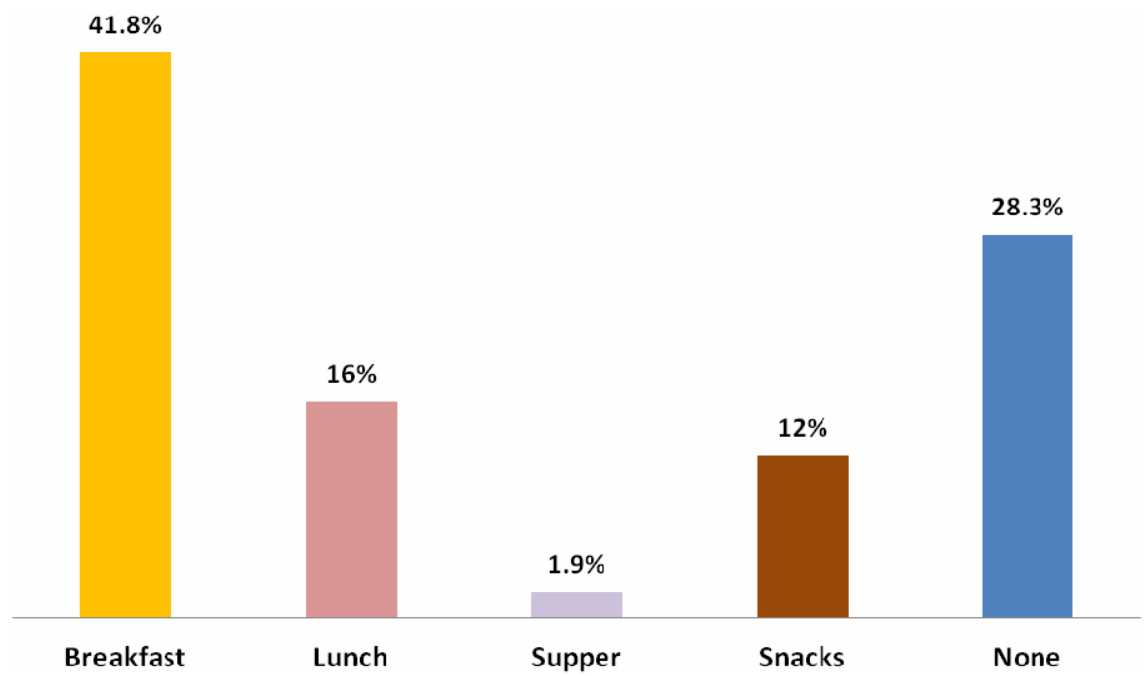

Figure 1. Meals frequently skipped by respondents. 
Table 5. Association between knowledge of diseases and dietary practices as measured by dietary diversity score (DDS).

\begin{tabular}{ccc}
\hline Knowledge of disease & Mean DDS & P value $^{*}$ \\
\hline Obesity & & 0.00 \\
Good & $4.00 \pm 0.01$ & \\
Fair & $2.15 \pm 0.84$ & \\
Poor & $1.19 \pm 0.13$ & \\
Diabetes & & 0.00 \\
Good & $4.00 \pm 0.01$ & \\
Fair & $2.85 \pm 0.05$ & \\
Poor & $1.10 \pm 0.07$ & \\
Hypertension & & \\
Good & $4.00 \pm 0.01$ & \\
Fair & $1.98 \pm 0.02$ & \\
Poor & $1.00 \pm 0.00$ & \\
\hline
\end{tabular}

*Association between knowledge of diseases and dietary diversity among respondents significant at $\mathrm{P}<0.01$.

\section{Conclusion and Recommendations}

The knowledge of diet-related chronic diseases in terms of the understanding, causes and prevention of diabetes, hypertension and obesity among day senior high school adolescents in the Ga-East Municipality was poor. Frequency of meal consumption was however good with most people consuming three meals a day. Breakfast was the most frequently skipped meal. Dietary diversity was generally low. A significant association was observed between knowledge of diseases and dietary diversity. As knowledge of the diseases increased, dietary diversity also improved. Based on the findings of the study, it is recommended that the Ghana Health Service nutrition education programs should target adolescents in Senior High Schools in order to increase their knowledge on diet-related chronic diseases. Again, the local name of hypertension influenced the respondents understanding of the disease. The Ghana Education Service should therefore include nutrition education in the curriculum of Senior High Schools to provide students with right information and advice, which will enable them to understand the diseases better so as eat healthy diets.

\section{REFERENCES}

[1] World Health Organization, "Diet, Nutrition and the Prevention of Chronic Diseases,” Report of WHO Study Group, Technical Report Series No. 797, Geneva, 2002.

[2] WHO, "Diet, Nutrition and Prevention of Communicable Diseases," 2005.

http://www.mcspotlight.org/media/reports/whorep.html”/r ecommendations
[3] Ghana Statistical Services and Macro International, "Ghana Demographic and Health Survey," Calverton, 2008.

[4] C. R. Berkley, "One Year Changes in Activity and Inactivity among 10-15 Years Old Boys and Girls: Relationship to Change in Body Mass Index," Pediatrics, Vol. 111, No. 4, 2003, pp. 836-843. doi:10.1542/peds.111.4.836

[5] B. Stewart and A. Tinsley, "From Qualitative to Quantitative: Development of an Instrument to Assess Food Choice Influences of Young Adults,” Ecology of Food and Nutrition, Vol. 34, No. 3, 1995, pp. 171-181. doi:10.1080/03670244.1995.9991458

[6] J. Lambert, C. Agostino, I. Elmadfa and K. Hulshof, "Dietary Intake and Nutritional Status in Children and Adolescents in Europe,” British Journal of Nutrition, Vol. 92, Suppl. 2, 2004, pp. 147S-211S. doi:10.1079/BJN20041160

[7] Center for Disease Control and Prevention, "Statistics on Diabetes, Hypertension and Obesity among Adolescents," Korle-Bu Teaching Hospital, Accra, 2009.

[8] W. B. Owusu and R. A. Nusenu, "Body Composition of Teenage Girls Using Bioelectrical Impedances Analysis,” Department of Nutrition and Food Science, University of Ghana, Legon, 2003.

[9] W. B. Owusu and E. Kwame, "Dietary Patterns, Lifestyle and Physical Development of Senior Secondary School Students in the Ho District of the Volta Region,” Department of Nutrition and Food Science, University of Ghana, Legon, 1998.

[10] I. A. Lansah, "Eating Habits of Adolescents at Madonna School, Koforidua,” A Dissertation Submitted to the Department of Home Science, University of Ghana, Legon, 2007.

[11] U. Ahmed and C. Del-Ninno, "Food for Education in Bangladesh: An Evaluation of Its Impact on Educational Attainment and Food Security,” International Food Research Institute, Bangladesh, 2002.

[12] P. Gleason and C. Suitor, "Food for Thought: Children diets in the 1990s,” Mathematics Policy Research Inc., Princeston, 2001.

[13] B. H. Lin, J. Guthrie and J. Blayblock, "The Diets of America's Children: Influences of Dinning out, Household Characteristics and Nutrition Knowledge,” US Department of Agriculture Economy Report 746, 1996.

[14] N. Kyei-Baffour, "Comparison of the Nutritional Status of Participating and Non Participating Pupils in the Ghana School Feeding Programme in Atwima Nwabiagya District of Ashanti Region of Ghana,” A Thesis Submitted to University of Ghana, Legon, 2009.

[15] M. Story and J. Stang, "Guidelines for Adolescent Nutrition Service," 2005. Http:/Www.Epi.Umn.Edu/Let/Pubs/Adol-BookShtm

[16] G. M. Wardlaw and P. M. Insel, "Perspective in Nutrition,” 3rd Edition, McGraw-Hill Company, New York, 1996. 